\title{
Tables
}

\section{CHAPTER 1: INTRODUCTION}

Table 1. Chimariko fieldwork in chronological order .7

Table 2. Different symbols used in Chimariko materials

\section{CHAPTER 2: PHONETICS AND PHONOLOGY}

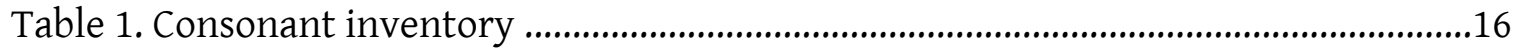

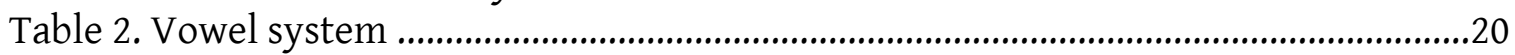

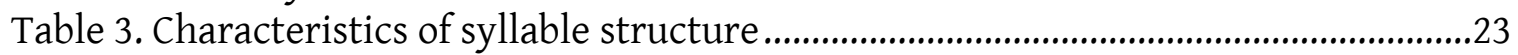

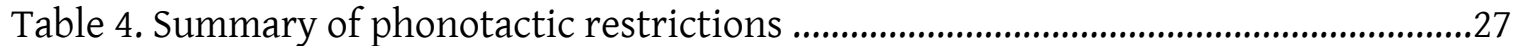

Table 5. Phonemic characteristics of Chimariko and its immediate neighbors ................29

Table 6. Characteristics of Chimariko and its distant neighbors to the (north-)west .....30

Table 7. Characteristics of Chimariko and its distant neighbors to the east........................30

Table 8. Characteristics of Northern California syllable structures ...................................31

\section{CHAPTER 3: MORPHOPHONEMIC ALTERNATIONS}

Table 1. Verb stem classes.

Table 2. Pronominal affixes for all verb stems...............................................................33

Table 3. Pronominal affix by verb stem class (following Harrington 020-1109) ................34

Table 4. Pronominal affixes including initial stem vowel by verb stem class ...................34

\section{CHAPTER 4: WORD CLASSES}

Table 1. Chimariko placenames (from Bauman 1980) ......................................................50

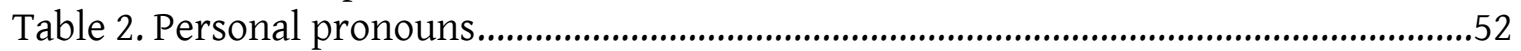

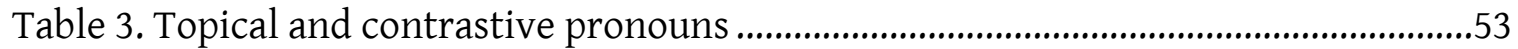

Table 4. Interrogative pronouns (Dixon, 1910:322; Harrington) ..........................................55

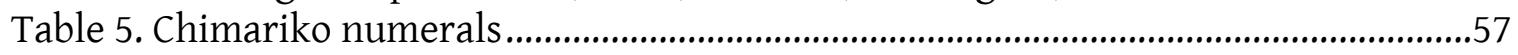

Table 6. Numeral systems in Northern California (from Haas 1976)...................................59

\section{CHAPTER 5: NOUN MORPHOLOGY}

Table 1. Possessive affixes

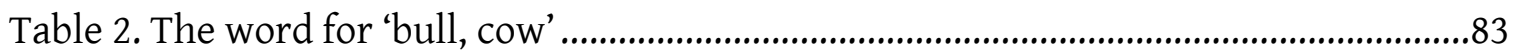

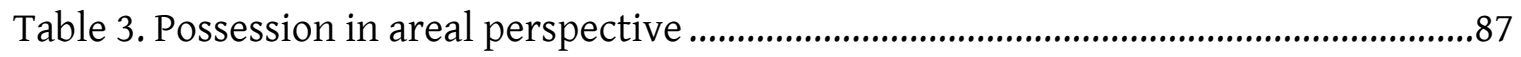

\section{CHAPTER 6: PRONOUN MORPHOLOGY}

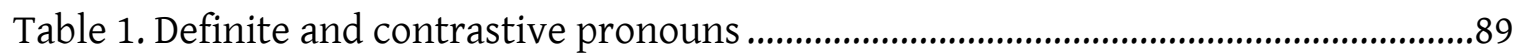

Table 2. Interrogative pronouns (Dixon, 1910:322; Harrington) .........................................91

Table 3. Pronoun shapes in Shasta and Chimariko ...........................................................93

\section{CHAPTER 8: VERB MORPHOLOGY}

Table 1. Verb templates for inflectional morphology ........................................................99

Table 2. Pronominal affixes including initial stem vowel by verb stem class .................100

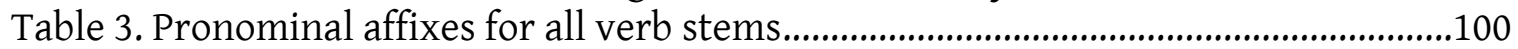

Table 4. Distinctions in bound pronominal marking ......................................................100 
Table 5. Person hierarchy in pronominal marking ...........................................................102

Table 6. Temporal suffixes .............................................................................................103

Table 7. Aspectual suffixes ....................................................................................................104

Table 8. Co-occurrence of -xana and -kon with other suffixes ...........................................106

Table 9. Modal affixes and clitics ...................................................................................111

Table 10. Conditional clauses..............................................................................................117

Table 11. Word formation with applicatives (from Grekoff 012.010) ..................................123

Table 12. Instrumental affixes (Dixon 1910:329) ..............................................................133

Table 13. Directional affixes...........................................................................................134

Table 14. Pronominal reference in Northern California ....................................................136

Table 15. Affixing pattern in tense/aspect/mood..............................................................137

Table 16. Reduplication in Northern California..................................................................138

Table 17. Directional and instrumental affixes.................................................................139

\section{CHAPTER 9: SIMPLE SENTENCES}

Table 1. Verb stems with first person patient markers (Grekoff 003.005)........................157

Table 2. Verb stems with first person agent OR patient markers (Grekoff 003.005).......158

\section{CHAPTER 10: QUESTIONS}

Table 1. Interrogative pronouns (Dixon, 1910:322; Harrington).......................................172

Table 2. Question formation strategies in Northern California ..........................................176

\section{CHAPTER 11: NEGATION}

Table 1. Morpheme templates with negative affixes ..........................................................178

Table 2. Negation strategies in Northern California ...........................................................183

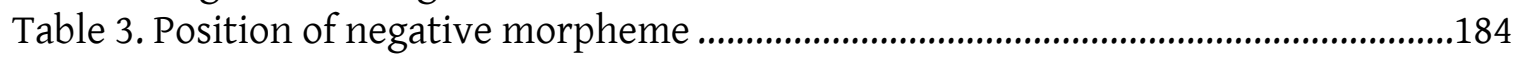

CHAPTER 12: COMPLEX SENTENCES

Table 1. Conditional clauses.........................................................................................196

\section{CHAPTER 14: SUMMARY}

Table 1. Similarities between Chimariko and its close neighbors and Karuk 